\title{
APPROXIMATION OF SOLUTIONS TO RETARDED DIFFERENTIAL EQUATIONS WITH APPLICATIONS TO POPULATION DYNAMICS
}

\author{
D. BAHUGUNA AND M. MUSLIM
}

Received 12 October 2003 and in revised form 24 July 2004

We consider a retarded differential equation with applications to population dynamics. We establish the convergence of a finite-dimensional approximations of a unique solution, the existence and uniqueness of which are also proved in the process.

\section{Introduction}

Consider the following partial differential equation with delay:

$$
\begin{gathered}
\frac{\partial u}{\partial t}(t, x)=\frac{\partial^{2} u}{\partial x^{2}}(t, x)+f(t, x, u(t, x), u(t-r, x)), \quad t>0, x \in[0,1], \\
\frac{\partial u}{\partial x}(t, 0)=0=\frac{\partial u}{\partial x}(t, 1), \quad t \geq 0, \\
u(s, x)=h(s, x), \quad s \in[-r, 0], x \in[0,1],
\end{gathered}
$$

where $f:[0, \infty) \times[0,1] \times \mathbb{R} \times \mathbb{R} \rightarrow \mathbb{R}$ and $h:[-r, 0] \times[0,1] \rightarrow \mathbb{R}$ is a given function. The above problem for $f(t, x, u, v)=-d(x) u+b(x) v$ models a linear growth of a population in $[0,1]$, where $u(t, \cdot)$ is the population density at time $t$, and the term $\partial^{2} u / \partial x^{2}$ represents the internal migration. The continuous functions $d, b:[0,1] \rightarrow[0, \infty)$ represent spacedependent death and birth rates, respectively, and $r$ is the delay due to pregnancy (cf. Engel and Nagel [10, page 434]).

We formulate (1.1) as the following retarded differential equation:

$$
\begin{gathered}
u^{\prime}(t)+A u(t)=f(t, u(t), u(a(t))), \quad 0<t \leq T<\infty, \\
u(t)=h(t), \quad t \in[-r, 0],
\end{gathered}
$$

in a Hilbert space $H$, where $-A$ is the infinitesimal generator of a $C_{0} \operatorname{semigroup}\{S(t): t \geq$ $0\}$ of bounded linear operators in $H, h \in \mathscr{C}_{0}:=C([-r, 0] ; H)$ is a given function and the function $a$ is defined from $[0, T]$ into $[-r, T]$ with the delay property $a(t) \leq t$ for $t \in[0, T]$. For (1.1), we may take $X=L^{2}[0,1]$ and $D(A)=\left\{u \in H^{2}[0,1]: u^{\prime}(0)=u^{\prime}(1)=0\right\}$ with $A u=-d^{2} u / d x^{2}$ for $u \in D(A)$. It is known that the semigroup $S(t)$ generated by $-A$ is analytic in $H$ (cf. Engel and Nagel [10, page 454]). 
For $t \in[0, T]$, we will use the notation $\mathscr{C}_{t}:=C([-r, t] ; H)$ for the Banach space of all continuous functions from $[-r, t]$ into $H$ endowed with the supremum norm

$$
\|\psi\|_{t}:=\sup _{-r \leq \eta \leq t}\|\psi(\eta)\|, \quad \psi \in \mathscr{C}_{t}
$$

The linear case of (1.2) in which $f(t, \psi)=L \psi$, with a bounded linear operator $L$ : $\mathscr{C}_{T} \rightarrow X$ is recently considered by Bátkai et al. [7] using the theory of perturbed HilleYosida operators. A particular semilinear case of (1.2) is considered by Alaoui [1].

For the earlier works on existence, uniqueness, and stability of various types of solutions of differential and functional differential equations, we refer to Bahuguna $[2,3]$, Balachandran and Chandrasekaran [6], Lin and Liu [13], and the references therein. The related results for the approximation of solutions may be found in $[4,5]$.

Initial studies concerning existence, uniqueness, and finite-time blowup of solutions for the equation

$$
\begin{gathered}
u^{\prime}(t)+A u(t)=g(u(t)), \quad t \geq 0 \\
u(0)=\phi
\end{gathered}
$$

have been considered by Segal [17], Murakami [15], and Heinz and von Wahl [12]. Bazley $[8,9]$ has considered the semilinear wave equation

$$
\begin{gathered}
u^{\prime \prime}(t)+A u(t)=g(u(t)), \quad t \geq 0, \\
u(0)=\phi, \quad u^{\prime}(0)=\psi
\end{gathered}
$$

and has established the uniform convergence of approximations of solutions to (1.5) using the existence results of Heinz and von Wahl [12]. Göthel [11] has proved the convergence of approximations of solutions to (1.4), but assumed $g$ to be defined on the whole of $H$. Based on the ideas of Bazley [8,9], Miletta [14] has proved the convergence of approximations to solutions of (1.4). The existence, uniqueness, and continuation of classical solutions to (1.2) are considered by Bahuguna [3]. In the present work, we use the ideas of Miletta [14] and Bahuguna [2, 3] to establish the convergence of finite-dimensional approximations of the solutions to (1.2).

\section{Preliminaries and assumptions}

Existence of a solution to (1.2) is closely associated with the existence of a function $u \in$ $\mathscr{C}_{\tilde{T}}, 0<\widetilde{T} \leq T$ satisfying

$$
u(t)= \begin{cases}h(t), & t \in[-r, 0], \\ S(t) h(0)+\int_{0}^{t} S(t-s) f(s, \psi(s), \psi(a(s))) d s, & t \in[0, \widetilde{T}]\end{cases}
$$

and such a function $u$ is called a mild solution of $(1.2)$ on $[-r, \widetilde{T}]$. A function $u \in \mathscr{C}_{\tilde{T}}$ is called a classical solution of (1.2) on $[-r, \tilde{T}]$ if $u \in C^{1}((0, \tilde{T}] ; H)$ and $u$ satisfies (1.2) on $[-r, \widetilde{T}]$. 
We assume that in (1.2), the linear operator $A$ satisfies the following hypothesis.

(H1) $A$ is a closed, positive definite, selfadjoint linear operator from the domain $D(A)$ $\subset H$ into $H$ such that $D(A)$ is dense in $H, A$ has the pure point spectrum

$$
0<\lambda_{0} \leq \lambda_{1} \leq \lambda_{2} \leq \cdots
$$

and a corresponding complete orthonormal system of eigenfunctions $\left\{u_{i}\right\}$, that is,

$$
A u_{i}=\lambda_{i} u_{i}, \quad\left(u_{i}, u_{j}\right)=\delta_{i j}
$$

where $\delta_{i j}=1$ if $i=j$ and zero otherwise.

If (H1) is satisfied, then the semigroup $S(t)$ generated by $-A$ is analytic in $H$. It follows that the fractional powers $A^{\alpha}$ of $A$ for $0 \leq \alpha \leq 1$ are well defined from $D\left(A^{\alpha}\right) \subseteq H$ into $H$ (cf. Pazy [16, pages 69-75]). $D\left(A^{\alpha}\right)$ is a Banach space endowed with the norm

$$
\|x\|_{\alpha}=\left\|A^{\alpha} x\right\|, \quad x \in D\left(A^{\alpha}\right) .
$$

For $t \in[0, T]$, we denote $\mathscr{C}_{t}^{\alpha}:=C\left([-r, t] ; D\left(A^{\alpha}\right)\right)$ endowed with the norm

$$
\|\psi\|_{t, \alpha}:=\sup _{-r \leq \eta \leq t}\|\psi(\eta)\|_{\alpha}
$$

The nonlinear function $f$ is assumed to satisfy the following hypotheses.

(H2) The function $h \in \mathscr{C}_{0}^{\alpha}$.

(H3) The map $f$ is defined from $[0, \infty) \times D\left(A^{\alpha}\right) \times D\left(A^{\alpha}\right)$ into $D\left(A^{\beta}\right)$ for $0<\beta \leq \alpha<1$ and there exists a nondecreasing function $L_{R}$ from $[0, \infty)$ into $[0, \infty)$ depending on $R>0$ such that

$$
\left\|f\left(t_{1}, u_{1}, v_{1}\right)-f\left(t_{2}, u_{2}, v_{2}\right)\right\| \leq L_{R}(t)\left[|t-s|^{\gamma}+\left\|u_{1}-u_{2}\right\|_{\alpha}+\left\|v_{1}-v_{2}\right\|_{\alpha}\right]
$$

for all $\left(t_{i}, u_{i}, v_{i}\right) \in[0, \infty) \times B_{R}\left(D\left(A^{\alpha}\right), h(0)\right) \times B_{R}\left(D\left(A^{\alpha}\right), h(a(0))\right)$, for $i=1,2$, where $0<$ $\gamma<1, B_{R}\left(Z, z_{0}\right)=\left\{z \in Z:\left\|z-z_{0}\right\|_{Z} \leq R\right\}$ is the ball of radius $R$ centered at $z_{0}$ in a Banach space $Z$ with its norm $\|\cdot\|_{Z}$.

(H4) The function $a:[0, T] \rightarrow[-r, T]$ is continuous and satisfies the delay property $a(t) \leq t$ for $t \in[0, T]$.

\section{Approximate solutions and convergence}

Let $H_{n}$ denote the finite-dimensional subspace of $H$ spanned by $\left\{u_{0}, u_{1}, \ldots, u_{n}\right\}$ and let $P^{n}: H \rightarrow H_{n}$ be the corresponding projection operator for $n=0,1,2, \ldots$ Let $0<t<\widetilde{T} \leq T$ be such that

$$
\begin{gathered}
\left\|(S(t)-I) A^{\alpha} h(0)\right\| \leq \frac{R}{3}, \\
\left\|A^{\alpha}\left(h_{n}(0)-h(0)\right)\right\| \leq \frac{R}{3} .
\end{gathered}
$$




\section{Retarded differential equations}

Let $\bar{h}$ be the extension of $h$ by the constant value $h(0)$ on $[0, T]$. We set

$$
T_{0}=\min \left\{\widetilde{T},\left(\frac{(1-\alpha) R}{3 M_{0} C_{\alpha}}\right)^{1 /(1-\alpha)},\left(\frac{3(1-\alpha)}{8 L_{R}\left(T_{0}\right) C_{\alpha}}\right)^{1 /(1-\alpha)}\right\}
$$

where $M_{0}=\left[L_{R}\left(T_{0}\right)\left(2 R+T^{\gamma}+4\|\bar{h}\|_{T_{0}, \alpha}\right)+\|f(0, h(0), h(a(0)))\|\right]$ and $C_{\alpha}$ is a positive constant such that $\left\|A^{\alpha} S(t)\right\| \leq C_{\alpha} t^{-\alpha}$ for $t>0$.

We define

$$
\begin{gathered}
f_{n}:\left[0, T_{0}\right] \times H \times H \longrightarrow D(A), \\
f_{n}(t, u, v)=P^{n} f\left(t, P^{n} u, P^{n} v\right), \quad(t, u, v) \in\left[0, T_{0}\right] \times H \times H, \\
h_{n}:[-r, 0] \longrightarrow D(A), \quad h_{n}(t)=P^{n} h(t), t \in[-r, 0] .
\end{gathered}
$$

Let $A^{\alpha}: \mathscr{C}_{t}^{\alpha} \rightarrow \mathscr{C}_{t}$ be given by $\left(A^{\alpha} \psi\right)(s)=A^{\alpha}(\psi(s)), s \in[-r, t], t \in\left[0, T_{0}\right]$. We define a $\operatorname{map} F_{n}: B_{R}\left(\mathscr{C}_{T_{0}}, A^{\alpha} \bar{h}\right) \rightarrow \mathscr{C}_{T_{0}}$ as follows:

$$
\left(F_{n} \psi\right)(t)= \begin{cases}A^{\alpha} h_{n}(t), & t \in[-r, 0], \\ S(t) A^{\alpha} h_{n}(0) & \\ +\int_{0}^{t} A^{\alpha} S(t-s) f_{n}\left(s, A^{-\alpha} \psi(s), A^{-\alpha} \psi(a(s))\right) d s, & t \in\left[0, T_{0}\right]\end{cases}
$$

for $\psi \in B_{R}\left(\mathscr{C}_{T_{0}}, A^{\alpha} \bar{h}\right)$.

Proposition 3.1. For each $n \geq n_{0}$, where $n_{0}$ is large enough and $n, n_{0} \in \mathbb{N}$, there exists a unique $w_{n} \in B_{R}\left(\mathscr{C}_{T_{0}}, A^{\alpha} \bar{h}\right)$ such that $F_{n} w_{n}=w_{n}$ on $\left[-r, T_{0}\right]$.

Proof. First, we show that for any $\psi \in B_{R}\left(\mathscr{C}_{T_{0}}, A^{\alpha} \bar{h}\right), F_{n} \psi \in B_{R}\left(\mathscr{C}_{T_{0}}, A^{\alpha} \bar{h}\right)$. For $t \in[-r, 0]$,

$$
\left(F_{n} \psi\right)(t)-A^{\alpha} \bar{h}(t)=A^{\alpha}\left(P^{n}-I\right) h(t)=A^{\alpha}\left(P^{n}-I\right) h(t) \longrightarrow 0, \quad \text { as } n \longrightarrow \infty .
$$

Thus, for $n \geq n_{0}, n_{0}$ large enough, for $t \in[-r, 0]$, we have

$$
\left\|\left(F_{n} \psi\right)(t)-A^{\alpha} \bar{h}(t)\right\| \leq R .
$$

Now, for $t \in\left(0, T_{0}\right]$, we have

$$
\begin{aligned}
\left\|\left(F_{n} \psi\right)(t)-A^{\alpha} \bar{h}(t)\right\| \leq & \left\|(S(t)-I) A^{\alpha} h(0)\right\|+\left\|A^{\alpha}\left(h_{n}(0)-h(0)\right)\right\| \\
& +\int_{0}^{t}\left\|A^{\alpha} S(t-s)\right\|\left\|f_{n}\left(s, A^{-\alpha} \psi(s), A^{-\alpha} \psi(a(s))\right)\right\| d s .
\end{aligned}
$$


For $s \in\left[0, T_{0}\right]$,

$$
\begin{aligned}
\| f_{n}(s, & \left.A^{-\alpha} \psi(s), A^{-\alpha} \psi(a(s))\right) \| \\
\leq & \left\|f\left(s, P^{n} A^{-\alpha} \psi(s), P^{n} A^{-\alpha} \psi(a(s))\right)\right\| \\
\leq & \left\|f\left(s, P^{n} A^{-\alpha} \psi(s), P^{n} A^{-\alpha} \psi(a(s))\right)-f\left(s, P^{n} \bar{h}(s), P^{n} \bar{h}(a(s))\right)\right\| \\
& +\left\|f\left(s, P^{n} \bar{h}(s), P^{n} \bar{h}(a(s))\right)-f(0, h(0), h(a(0)))\right\| \\
& +\|f(0, h(0), h(a(0)))\| \\
\leq & L_{R}\left(T_{0}\right)\left(2 R+T^{\gamma}+4\|\bar{h}\|_{T_{0}, \alpha}\right)+\|f(0, h(0), h(a(0)))\| .
\end{aligned}
$$

It follows from the choice of $T_{0}$ that $F_{n}: B_{R}\left(\mathscr{C}_{T_{0}}, A^{\alpha} \bar{h}\right) \rightarrow B_{R}\left(\mathscr{C}_{T_{0}}, A^{\alpha} \bar{h}\right)$ for $n$ large enough. Now, we show that $F_{n}$ is a strict contraction. For $\psi_{1}, \psi_{2} \in B_{R}\left(\mathscr{C}_{T_{0}}, A^{\alpha} \bar{h}\right),\left(F_{n} \psi_{1}\right)(t)-$ $\left(F_{n} \psi_{2}\right)(t)=0$ on $[-r, 0]$ and for $t \in\left[0, T_{0}\right]$, we have

$$
\left\|\left(F_{n} \psi_{1}\right)(t)-\left(F_{n} \psi_{2}\right)(t)\right\| \leq 2 L_{R}\left(T_{0}\right) C_{\alpha} \frac{T_{0}^{1-\alpha}}{1-\alpha}\left\|\psi_{1}-\psi_{2}\right\|_{T_{0}} \leq \frac{3}{4}\left\|\psi_{1}-\psi_{2}\right\|_{T_{0}} .
$$

Taking the supremum over $\left[-r, T_{0}\right]$, it follows that $F_{n}$ is a strict contraction on $B_{R}\left(\mathscr{C}_{T_{0}}\right.$, $\left.A^{\alpha} \bar{h}\right)$ and hence there exits a unique $w_{n} \in B_{R}\left(\mathscr{C}_{T_{0}}, A^{\alpha} \bar{h}\right)$ with $w_{n}=F_{n} w_{n}$ on $\left[-r, T_{0}\right]$. This completes the proof of the proposition.

Let $u_{n}=A^{-\alpha} w_{n}$. Then, $u_{n} \in B_{R}\left(\mathscr{C}_{T_{0}}^{\alpha}, \bar{h}\right)$ and satisfies

$$
u_{n}(t)= \begin{cases}h_{n}(t), & t \in[-r, 0] \\ S(t) h_{n}(0) & \\ +\int_{0}^{t} S(t-s) f_{n}\left(s, u_{n}(s), u_{n}(a(s))\right) d s, & t \in\left[0, T_{0}\right]\end{cases}
$$

Remarks 3.2. The above solution $u_{n}(t)$ is known as the Faedo-Galerkin approximate solution of (1.2).

Collorary 3.3. If $h(t) \in D(A)$ for all $t \in[-\tau, 0]$, then $w_{n}(t) \in D\left(A^{\beta}\right)$ for all $t \in\left[-\tau, T_{0}\right]$, where $0 \leq \beta<1,0 \leq \alpha+\beta<1$, and $w_{n}(t)$ is the solution of the integral equation (3.4).

Proof. For any $g \in D\left(A^{\beta}\right)$ and $t \in[-\tau, 0]$, we have

$$
\left|\left(A^{\beta} g, w_{n}(t)\right)\right| \leq\|g\| \| A^{\beta+\alpha} h_{n}(t)|| .
$$

Now, for any $t \in\left(0, T_{0}\right]$, we have

$$
\begin{aligned}
\left(A^{\beta} g, w_{n}(t)\right)= & \left(g, A^{\beta+\alpha} S(t) h_{n}(0)\right) \\
& +\int_{0}^{t}\left(g, A^{\beta+\alpha} S(t-s), f_{n}\left(s, A^{-\alpha} w_{n}(s), A^{-\alpha} w_{n}(a(s))\right)\right) d s .
\end{aligned}
$$

The first term is bounded for $t \in(0, T]$ as

$$
\left|\left(A^{\beta} g, S(t) h_{n}(0)\right)\right| \leq\|g\| M\left\|A^{\beta+\alpha} h(0)\right\| .
$$


The second term is treated as follows:

$$
\left\|\int_{0}^{t}\left(g, A^{\beta+\alpha} S(t-s), f_{n}\left(s, u_{n}(s), u_{n}(a(s))\right)\right) d s\right\| \leq M_{0}\|g\| C_{\beta+\alpha} \frac{T_{0}^{1-(\beta+\alpha)}}{1-(\beta+\alpha)} .
$$

Hence the corollary is proved.

Collorary 3.4. If $h(t) \in D(A)$ for all $t \in[-\tau, 0]$, then for any $t \in\left[-\tau, T_{0}\right]$, there exists a constant $M_{1}$, independent of $n$, such that

$$
\left\|A^{\beta} w_{n}(t)\right\| \leq M_{0}
$$

for all $-\tau \leq t \leq T_{0}$ and $0 \leq \beta<1$.

Corollary 3.4 is a consequence of Corollary 3.3.

Proposition 3.5. The sequence $\left\{u_{n}\right\} \subset \mathscr{C}_{T_{0}}$ is a Cauchy sequence and therefore converges to a function $u \in \mathscr{C}_{T_{0}}$ if the assumptions (H1)-(H4) hold.

Proof. From Proposition 3.1 we have (3.10). With $u_{n}=A^{-\alpha} w_{n}$, (3.10) becomes

$$
w_{n}(t)= \begin{cases}A^{\alpha} h_{n}(t), & t \in[-r, 0], \\ S(t) A^{\alpha} h_{n}(0) & \\ +\int_{0}^{t} A^{\alpha} S(t-s) f_{n}\left(s, A^{-\alpha} u_{n}(s), A^{-\alpha} u_{n}(a(s))\right) d s, & t \in\left[0, T_{0}\right] .\end{cases}
$$

For $n \geq m \geq n_{0}$, where $n_{0}$ is large enough, $n, m, n_{0} \in \mathbb{N}, t \in[-r, 0]$, we have

$$
\begin{aligned}
\left\|w_{n}(t)-w_{m}(t)\right\| & \leq\left\|h_{n}(t)-h_{m}(t)\right\|_{\alpha} \\
& \leq\left\|\left(P^{n}-P^{m}\right) h(t)\right\|_{\alpha} \longrightarrow 0 \quad \text { as } m \longrightarrow \infty .
\end{aligned}
$$

For $t \in\left(0, T_{0}\right]$ and $n, m$, and $n_{0}$ as above, we have

$$
\begin{aligned}
\left\|w_{n}(t)-w_{m}(t)\right\| \leq & \left\|\left(P^{n}-P^{m}\right) S(t) A^{\alpha} h(0)\right\| \\
+\int_{0}^{t} \| A^{\alpha} S(t-s)[ & f_{n}\left(s, A^{-\alpha} w_{n}(s), A^{-\alpha} w_{n}(a(s))\right) \\
& \left.\quad-f_{m}\left(s, A^{-\alpha} w_{m}(s), A^{-\alpha} w_{m}(a(s))\right)\right] \| d s .
\end{aligned}
$$

Now, using Corollaries 3.3 and 3.4, we have

$$
\begin{aligned}
&\left\|f_{n}\left(s, A^{-\alpha} w_{n}(s), A^{-\alpha} w_{n}(a(s))-f_{m}\left(s, A^{-\alpha} w_{m}(s), A^{-\alpha} w_{m}(a(s))\right)\right)\right\| \\
& \leq\left\|\left(P^{n}-P^{m}\right) f\left(s, P^{m} A^{-\alpha} w_{m}(s), P^{m} A^{-\alpha} w_{m}(a(s))\right)\right\| \\
&+L_{R}\left(T_{0}\right)\left[\left\|\left(P^{n}-P^{m}\right) w_{m}(s)\right\|+\left\|\left(P^{m}-P^{m}\right) w_{m}(a(s))\right\|\right] \\
&+2 L_{R}\left(T_{0}\right)\left\|w_{n}-w_{m}\right\|_{s} \\
& \leq C_{1}+C_{2} \frac{1}{\lambda_{m}^{\beta}}+C_{2}\left\|w_{n}-w_{m}\right\|_{s}
\end{aligned}
$$


for some positive constants $C_{1}$ and $C_{2}$, where $C_{1}=\left\|\left(p^{n}-P^{m}\right)\right\|\left[L_{R}\left(T_{0}\right)\left(2 R+T^{\gamma}+\right.\right.$ $\left.\left.4\|\bar{h}\|_{T_{0}, \alpha}\right)+\|f(0, h(0), h(a(0)))\|\right]$ and $C_{2}=2 L_{R}\left(T_{0}\right)$. Thus, we have the following estimate:

$$
\begin{aligned}
\left\|w_{n}(t)-w_{m}(t)\right\| \leq & C_{0}\left\|\left(P^{n}-P^{m}\right) A^{\alpha} h(0)\right\|+\frac{C_{1} C_{\alpha} T_{0}^{1-\alpha}}{(1-\alpha)}+\frac{C_{2} C_{\alpha} T_{0}^{1-\alpha}}{(1-\alpha) \lambda_{m}^{\beta}} \\
& +C_{2} C_{\alpha} \int_{0}^{t}(t-s)^{-\alpha}\left\|w_{n}-w_{m}\right\|_{s} d s
\end{aligned}
$$

where $C_{0}=M e^{\omega T}$. Since $\left\|w_{n}-w_{m}\right\|=\left\|h_{n}-h_{m}\right\|_{\alpha}$ on $[-r, 0]$, we have

$$
\begin{aligned}
\left\|w_{n}-w_{m}\right\|_{t} \leq & \left\|h_{n}-h_{m}\right\|_{\alpha}+C_{0}\left\|\left(P^{n}-P^{m}\right) A^{\alpha} h(0)\right\|+\frac{C_{1} C_{\alpha} T_{0}^{1-\alpha}}{(1-\alpha)} \\
& +\frac{C_{2} C_{\alpha} T_{0}^{1-\alpha}}{(1-\alpha) \lambda_{m}^{\beta}}+C_{2} C_{\alpha} \int_{0}^{t}(t-s)^{-\alpha}\left\|w_{n}-w_{m}\right\|_{s} d s .
\end{aligned}
$$

Application of Gronwall's inequality gives the required result. This completes the proof of the proposition.

With the help of Propositions 3.1 and 3.5, we may state the following existence, uniqueness, and convergence result.

Theorem 3.6. Suppose that (H1)-(H4) hold. Then, there exist unique functions $u_{n} \in$ $C\left(\left[-r, T_{0}\right] ; H_{n}\right)$ and $u \in C\left(\left[-r, T_{0}\right] ; H\right)$ satisfying (3.10) and

$$
u(t)= \begin{cases}h(t), & t \in[-r, 0], \\ S(t) h(0) & \\ +\int_{0}^{t} S(t-s) f(s, u(s), u(a(s))) d s, & t \in\left[0, T_{0}\right],\end{cases}
$$

such that $u_{n} \rightarrow u$ in $C\left(\left[-r, T_{0}\right] ; H\right)$ as $n \rightarrow \infty$, where $h_{n}(t)=P^{n} h(t)$ and $f_{n}(t, u, v)=$ $P^{n} f\left(t, P^{n} u, P^{n} v\right)$.

\section{Regularity}

The functions $u_{n}$ and $u$ in Theorem 3.6 satisfying (3.10) and (3.22) may be called approximate mild solution and mild solution of (1.2) on $\left[-\tau, T_{0}\right]$, respectively. In this section, we establish the regularity of the mild solution $u$ of (1.2) under an additional assumption of Hölder continuity of the function $a$ on $[0, T]$. We note that if $a$ is Lipschitz continuous on $[0, T]$, then it is also Hölder continuous on $[0, T]$. We establish the following regularity result.

Theorem 4.1. Suppose that (H1)-(H4) hold and, in addition, suppose that $a:[0, T] \rightarrow$ $[-r, T]$ is Hölder continuous, that is, there exist constants $0<\delta<1$ and $L_{a} \geq 0$ such that

$$
|a(t)-a(s)| \leq L_{a}|t-s|^{\delta} .
$$

Then, the mild solution $u$ given by (3.10) of (1.2) is a unique classical solution of (1.2) on $\left[-r, T_{0}\right]$. 


\section{Retarded differential equations}

We prove that $u$ is in fact a unique classical solution. For this, we first prove that the mild solution

$$
u(t)= \begin{cases}h(t), & t \in[-r, 0], \\ S(t) h(0)+\int_{0}^{t} S(t-s) f(s, u(s), u(a(s))) d s, & t \in\left[0, T_{0}\right]\end{cases}
$$

is locally Hölder continuous on $(0, \tilde{T}]$. Let $v(t)=A^{\alpha} u(t)$. Then,

$$
v(t)= \begin{cases}A^{\alpha} h(t), & t \in[-r, 0], \\ S(t) A^{\alpha} h(0)+\int_{0}^{t} S(t-s) A^{\alpha} f\left(s, A^{-\alpha} v(s), A^{-\alpha} v(a(s))\right) d s, & t \in\left[0, T_{0}\right] .\end{cases}
$$

Let

$$
N=\sup _{t \in[0, \widetilde{T}]}\left\|f\left(t, A^{-\alpha} v(t), A^{-\alpha} v(a(t))\right)\right\| .
$$

It is known that (cf. [16, page 197]) for every $\beta$ with $0<\beta<1-\alpha$ and every $0<h<1$, we have

$$
\left\|(S(h)-I) A^{\alpha} S(t-s)\right\| \leq C_{\beta} h^{\beta}\left\|A^{\alpha+\beta} S(t-s)\right\| \leq C h^{\beta}(t-s)^{-\alpha+\beta}, \quad 0<s<t .
$$

For $0<t<t+h \leq T_{0}$, we have

$$
\begin{aligned}
\|v(t+h)-v(t)\| \leq & \left\|(S(h)-I) A^{\alpha} S(t) \chi(0)\right\| \\
& +\int_{0}^{t}\left\|(S(h)-I) A^{\alpha} S(t-s) f\left(s, A^{-\alpha} v(s), A^{-\alpha} v(a(s))\right)\right\| d s \\
& +\int_{t}^{t+h}\left\|A^{\alpha} S(t+h-s) f\left(s, A^{-\alpha} v(s), A^{-\alpha} v(a(s))\right)\right\| d s .
\end{aligned}
$$

Using (4.5), we get

$$
\left\|(S(h)-I) A^{\alpha} S(t) \chi(0)\right\| \leq C t^{-(\alpha+\beta)} h^{\beta}\|\chi(0)\| \leq M_{1} h^{\beta},
$$

where $M_{1}$ depends on $t$ and $M_{1} \rightarrow \infty$ as $t \rightarrow 0$. Now,

$$
\begin{aligned}
\int_{0}^{t} \|( & (h)-I) A^{\alpha} S(t-s) f\left(s, A^{-\alpha} v(s), A^{-\alpha} v(a(s))\right) \| d s \\
& \leq C N h^{\beta} \int_{0}^{t}(t-s)^{-(\alpha+\beta)} d s \\
& \leq M_{2} h^{\beta}
\end{aligned}
$$


where $M_{2}$ is independent of $t$. For the last integral in (4.6), we have

$$
\begin{aligned}
\int_{t}^{t+h} & \left\|A^{\alpha} S(t+h-s) f\left(s, A^{-\alpha} v(s), A^{-\alpha} v(a(s))\right)\right\| d s \\
& \leq N C_{\alpha} \int_{t}^{t+h}(t+h-s)^{-\alpha} d s \\
& \leq \frac{N C_{\alpha}}{1-\alpha} h^{1-\alpha} \\
& \leq M_{3} h^{\beta}
\end{aligned}
$$

where $M_{3}$ is also independent of $t$. The above estimates imply that

$$
|| v(t)-v(s)|| \leq L_{v}|t-s|^{\beta}, \quad|t-s|<1,0<s, t \leq T_{0} .
$$

For any $0<s<t \leq T_{0}$, with $t-s \geq 1$, we insert $t_{1}<t_{2}<\cdots<t_{n}$ between $s$ and $t$ such that $1 / 2 \leq t_{i+1}-t_{i}<1$ for $i=1,2, \ldots, n-1$ and $t-t_{n}<1$. Clearly, $n \leq 2 T_{0} \leq 2 T$. Then, for $0<s<t \leq T_{0}$, with $t-s \geq 1$, we have

$$
\begin{aligned}
\|v(t)-v(s)\| & \leq\left\|v(t)-v\left(t_{n}\right)\right\|+\sum_{i=1}^{n-1}\left\|v\left(t_{i+1}\right)-v\left(t_{i}\right)\right\|+\left\|v\left(t_{1}\right)-v(s)\right\| \\
& \leq L_{v}\left[\left(t-t_{n}\right)^{\beta}+\sum_{i=1}^{n-1}\left(t_{i+1}-t_{i}\right)^{\beta}+\left(t_{1}-s\right)^{\beta}\right] \\
& \leq(2 T+1) L_{v}|t-s|^{\beta}=\widetilde{L}_{v}|t-s|^{\beta},
\end{aligned}
$$

where $\tilde{L}_{v}=(2 T+1) L_{v}$. Now, for $0<s, t \leq T_{0}$, we have

$$
\begin{aligned}
\| f(t & \left.A^{-\alpha} v(t), A^{-\alpha} v(a(t))\right)-f\left(s, A^{-\alpha} v(s), A^{-\alpha} v(a(s))\right) \| \\
& \leq L_{f}(R)\left[|t-s|^{\gamma}+\|v(t)-v(s)\|+\|v(a(t))-v(a(s))\|\right] \\
& \leq L_{f}(R)\left[|t-s|^{\gamma}+\widetilde{L}_{v}|t-s|^{\beta}+\widetilde{L}_{v}|a(t)-a(s)|^{\beta}\right] \\
& \leq L_{f}(R)\left[|t-s|^{\gamma}+\widetilde{L}_{v}|t-s|^{\beta}+\widetilde{L}_{v} L_{a}^{\beta}|t-s|^{\delta \cdot \beta}\right] \\
& \leq L_{f}(R)\left(1+\widetilde{L}_{v}+\widetilde{L}_{v} L_{a}^{\beta}\right)|t-s|^{\max \{\gamma, \beta, \delta \cdot \beta\}},
\end{aligned}
$$

which shows that the function $t \mapsto f\left(t, A^{-\alpha} v(t), A^{-\alpha} v(a(t))\right)$ is locally Hölder continuous on $\left(0, T_{0}\right]$.

Now, consider the initial value problem

$$
\frac{d w(t)}{d t}+A w(t)=f\left(t, A^{-\alpha} v(t), A^{-\alpha} v(a(t))\right), \quad w(0)=\chi(0)
$$


By [16, Corollary 4.3.3], (4.13) has a unique solution $w \in C^{1}\left(\left(0, T_{0}\right] ; H\right)$ given by

$$
w(t)=S(t) \chi(0)+\int_{0}^{t} S(t-s) f\left(s, A^{-\alpha} v(s), A^{-\alpha} v(a(s))\right) d s .
$$

For $t>0$, each term on the right of (4.14) is in $D(A) \subseteq D\left(A^{\alpha}\right)$, we may apply $A^{\alpha}$ on $w$ to get

$$
A^{\alpha} w(t)=S(t) A^{\alpha} \chi(0)+\int_{0}^{t} A^{\alpha} S(t-s) f\left(s, A^{-\alpha} v(s), A^{-\alpha} v(a(s))\right) d s
$$

The right-hand side of (4.15) is equal to $v(t)$ and therefore $w(t)=u(t)$ on $\left[0, T_{0}\right]$. Thus, $u \in C^{1}\left(\left(0, T_{0}\right] ; H\right)$ and hence $u$ is a classical solution of $(1.2)$. This completes the proof of the theorem.

\section{Acknowledgment}

The authors would like to thank the National Board for Higher Mathematics for providing the financial support to carry out this work under its research project no. NBHM/ 2001/ R\&D-II.

\section{References}

[1] L. Alaoui, Nonlinear homogeneous retarded differential equations and population dynamics via translation semigroups, Semigroup Forum 63 (2001), no. 3, 330-356.

[2] D. Bahuguna, Existence, uniqueness and regularity of solutions to semilinear nonlocal functional differential problems, Nonlinear Anal. 57 (2004), no. 7-8, 1021-1028.

[3] Existence, uniqueness, and regularity of solutions to semilinear retarded differential equations, J. Appl. Math. Stochastic Anal. 2004 (2004), no. 3, 213-219.

[4] D. Bahuguna and R. Shukla, Approximations of solutions to second order semilinear integrodifferential equations, Numer. Funct. Anal. Optim. 24 (2003), no. 3-4, 365-390.

[5] D. Bahuguna, S. K. Srivastava, and S. Singh, Approximations of solutions to semilinear integrodifferential equations, Numer. Funct. Anal. Optim. 22 (2001), no. 5-6, 487-504.

[6] K. Balachandran and M. Chandrasekaran, Existence of solutions of a delay differential equation with nonlocal condition, Indian J. Pure Appl. Math. 27 (1996), no. 5, 443-449.

[7] A. Bátkai, L. Maniar, and A. Rhandi, Regularity properties of perturbed Hille-Yosida operators and retarded differential equations, Semigroup Forum 64 (2002), no. 1, 55-70.

[8] N. W. Bazley, Approximation of wave equations with reproducing nonlinearities, Nonlinear Anal. 3 (1979), no. 4, 539-546.

[9] - Global convergence of Faedo-Galerkin approximations to nonlinear wave equations, Nonlinear Anal. 4 (1980), no. 3, 503-507.

[10] K.-J. Engel and R. Nagel, One-Parameter Semigroups for Linear Evolution Equations, Graduate Texts in Mathematics, vol. 194, Springer-Verlag, New York, 2000.

[11] R. Göthel, Faedo-Galerkin approximations in equations of evolution, Math. Methods Appl. Sci. 6 (1984), no. 1, 41-54.

[12] E. Heinz and W. von Wahl, Zu einem Satz von F. E. Browder über nichtlineare Wellengleichungen, Math. Z. 141 (1975), 33-45 (German).

[13] Y. P. Lin and J. H. Liu, Semilinear integrodifferential equations with nonlocal Cauchy problem, Nonlinear Anal. 26 (1996), no. 5, 1023-1033.

[14] P. D. Miletta, Approximation of solutions to evolution equations, Math. Methods Appl. Sci. 17 (1994), no. 10, 753-763. 
[15] H. Murakami, On non-linear ordinary and evolution equations, Funkcial. Ekvac. 9 (1966), 151162.

[16] A. Pazy, Semigroups of Linear Operators and Applications to Partial Differential Equations, Applied Mathematical Sciences, vol. 44, Springer-Verlag, New York, 1983.

[17] I. Segal, Non-linear semi-groups, Ann. of Math. (2) 78 (1963), 339-364.

D. Bahuguna: Department of Mathematics and Statistics, Indian Institute of Technology, Kanpur 208 016, India

E-mail address: dhiren@iitk.ac.in

M. Muslim: Department of Mathematics and Statistics, Indian Institute of Technology, Kanpur 208 016, India

E-mail address: muslim@iitk.ac.in 


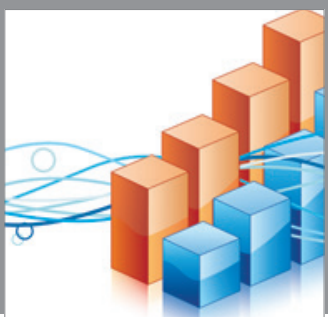

Advances in

Operations Research

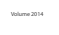

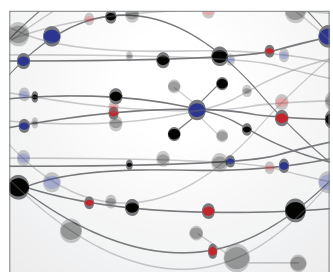

\section{The Scientific} World Journal
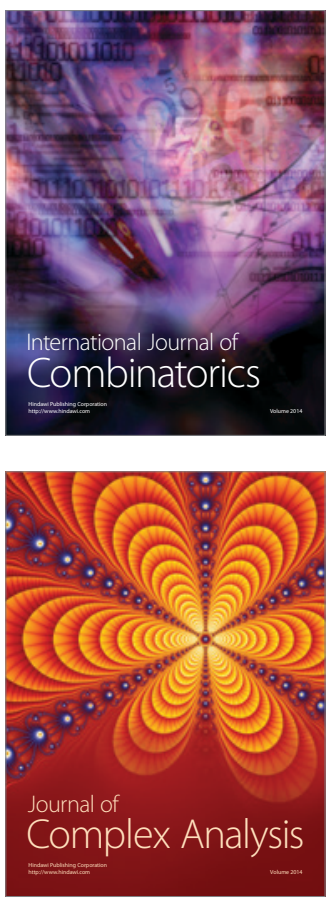

International Journal of

Mathematics and

Mathematical

Sciences
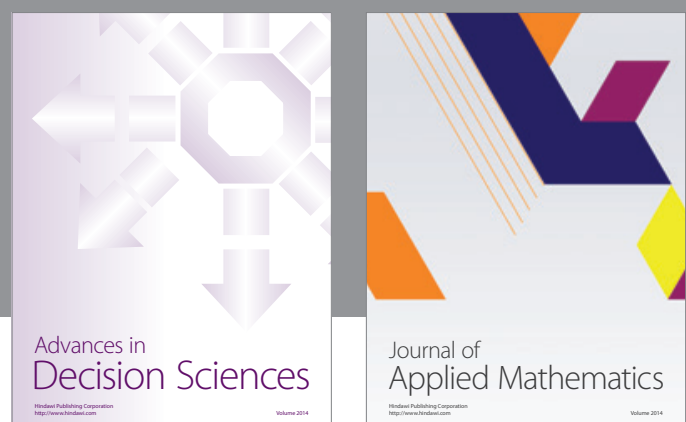

Journal of

Applied Mathematics
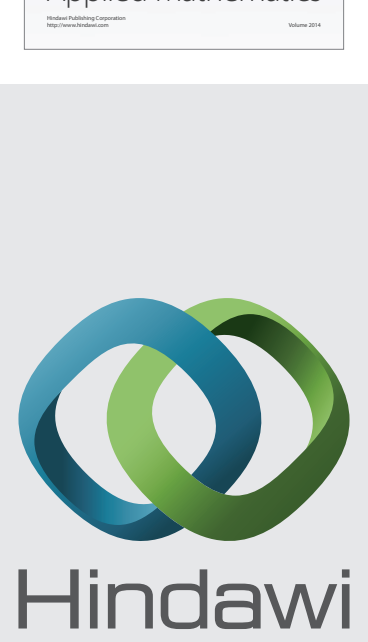

Submit your manuscripts at http://www.hindawi.com
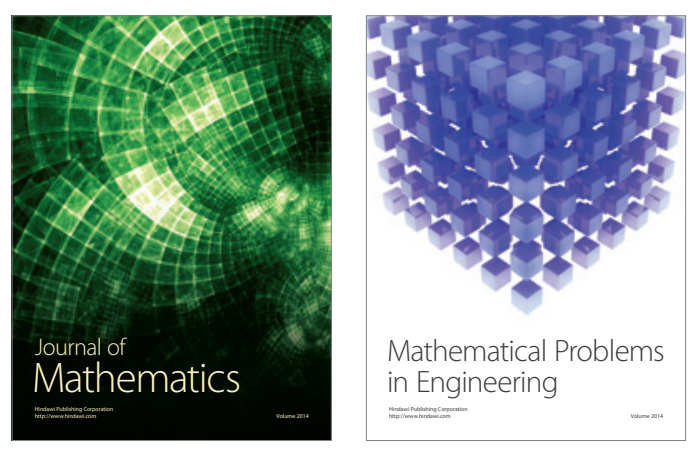

Mathematical Problems in Engineering
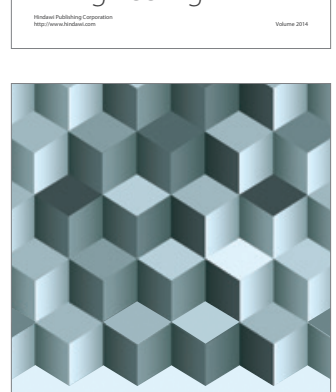

Journal of

Function Spaces
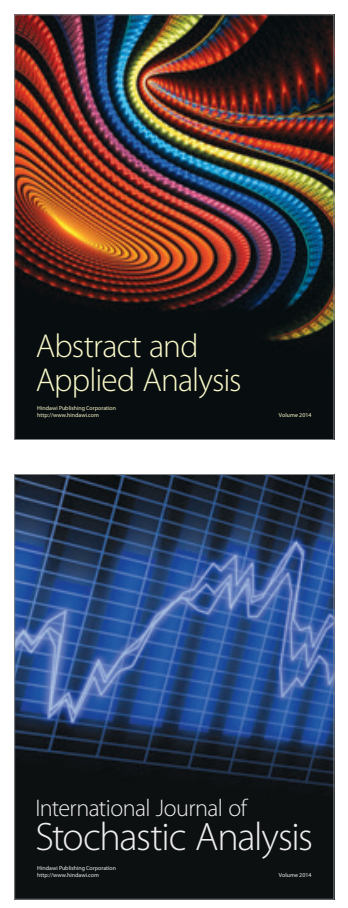

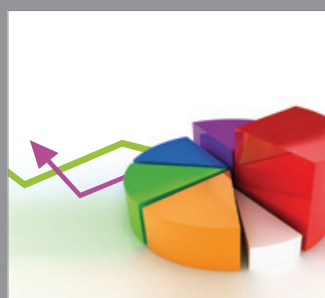

ournal of

Probability and Statistics

Promensencen
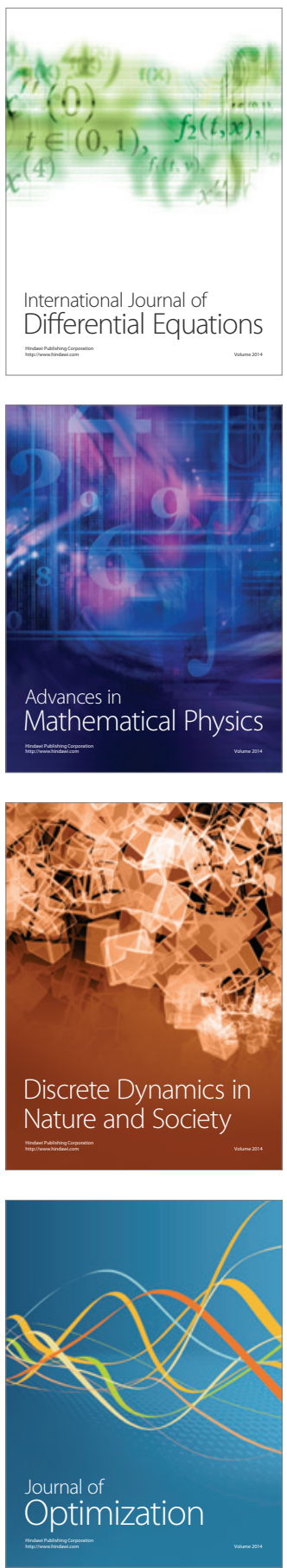